\title{
PARALYTIC ILEUS
}

\author{
By L. P. Le Quesne, D.M., F.R.C.S. \\ Department of Surgical Studies, The Middlesex Hospital
}

Established paralytic ileus, with gross distension and severe water and electrolyte depletion, is no longer a common post-operative complication. Nonetheless, bearing in mind those cases of frank ileus that still occur, and all those patients who develop ' wind ' to a greater or lesser extent after operation, it is apparent that the problem of the return of normal intestinal motility after operation is one that demands conscious attention. Of greatest importance after abdominal operations, the problem is not confined to this branch of surgery, for ileus may develop after operations on the urinary system, on the female pelvic organs, and on the spine, and rarely following injury to the spine or abdominal organs or as a complication during the administration of certain drugs (e.g. mecamylamine hydrochloride).

For many years the clinical features and pathological changes in paralytic ileus have been well recognized, but it is only comparatively recently that its aetiology has been understood and that it has been possible to plan rational prophylaxis and treatment. To a considerable extend the confusion surrounding the condition has stemmed from its misleading name. Paralytic ileus is, in fact, a functional obstruction of the intestines, with marked distension, usually of the entire small and large gut, by gas and fluid. Accordingly, the two main questions to be faced are: (I) Why is there a functional obstruction? (2) Why are the intestines distended? The statement of the problem in this form is important: in the past a failure to do so has led to a number of theories which postulate a flaccid paralysis of the intestines, resulting in no clearly defined way in a passive distension of the gut. But set out in this form much of the mystery is removed and replaced by two relatively clear-cut questions.

\section{Aetiology \\ The Functional Obstruction}

After every abdominal operation there is an inhibition of intestinal motility which lasts for a period which is in general in direct proportion to the severity of the operation. Peritonitis is accompanied by a similar inhibition which relents as the

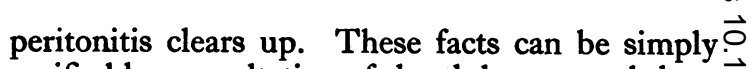
verified by auscultation of the abdomen, and there $\vec{\omega}$ is, in addition, experimental evidence to the sameo effect. Wakim and Mann (1943) carried out ex-70 periments on dogs with exteriorized loops of in -3 . testine and showed that after a laparotomy under $\dot{\omega}_{\tilde{\omega}}^{\mathrm{c}}$ general anaesthesia there was complete abolition $\omega$ of intestinal activity for at least four hours and $\mathscr{\infty}^{\infty}$ that normal activity did not return for 24 hours, 8 whilst after an operation involving division and anastomosis of the intestines activity was abolished음 for 42 hours, after which peristalsis slowly returned and regained its normal activity by the sixth day. Information about the activity of the human gut $\varnothing_{\mathbb{Q}}$ after operation is not so complete, but Bisgard and 3 Johnson (1939), by recording pressure changes In swallowed balloons, have shown a diminution fir intestinal motility after operation, and Davis ang Hansen (1945) demonstrated that barium traverse the intestine more slowly after operation than $\tilde{i n}_{0}$ normal circumstances. Devine (1946) has also shown, by the use of intestinal balloons, thato intestinal motility is inhibited after operation and $\frac{\mathscr{Q}}{\not}$ that on their return contractions are at first $\stackrel{\circ}{\circ}$ irregular before the re-establishment of normal $\vec{O}$ peristalsis.

This post-operative diminution in intestinal motility is not due to an actual paralysis of the gut? musculature, but to a reflex inhibition of its응 activity. Speaking in general terms, peristalsis is an inherent property of the intestines dependent $\frac{3}{3}$ upon the intramural nerve plexuses, with the extrinsic sympathetic and para-sympathetic nerves $\frac{0}{3}$ only exercising a controlling influence. Thus peristalsis can occur in a denervated loop of intestine,,$\frac{9}{5}$ and in a normally innervated loop stimulation of $D$ the para-sympathetic supply increases peristaltic activity whilst sympathetic stimulation depresses $N$ or completely inhibits muscular contractions. The depression of gut motility occurring after operation $\mathrm{N}$ is not due to any interference with the intrinsic $\omega$ mechanism of the intestine, but is a reflex phenomenon mediated via the sympathetic and can beco temporarily abolished by paralysis of the sym- $\mathbb{\Phi}$ pathetic nerves. If a spinal anaesthetic is given to a patient during the period of absent peristalsis, $\frac{T}{0}$ 
active gut movements immediately return and flatus may be expelled. This must mean that the absence of peristalsis is not an intrinsic condition, but is reflexly imposed on the bowel. The afferent impulses presumably arise as a result of irritation of the peritoneum, or from emotional stimuli (ileus is more common in nervous than placid patients), or from direct irritation of the nerves, thus accounting for the inhibition after injuries or operations on the spine and retroperitoneal structures (i.e. kidney).

A period of reflex inhibition of intestinal motility is then a normal occurrence after all abdominal operations and may occur after various other procedures, and it is only when distension is superadded that so-called paralytic ileus develops. It is important to remember that the 'paralytic' feature of the condition is a normal occurrence after many operations, so that the crucial problem becomes, why do some patients, in addition, develop distension, causing either ' wind' or, in severe cases, full-blown paralytic ileus.

\section{The Cause of Distension}

The answer to the problem is to be found in a consideration of the nature of the distension. The fluid in the distended gut consists of intestinal juices and it is the gas which forms the crux of the problem. There are three possible ways in which gas can accumulate in the bowel, namely, by decomposition of intestinal contents, by diffusion from the blood gases, and by the swallowing of air. It appears that in paralytic ileus (i.e. functional obstruction), as in cases of organic obstruction, the last of these routes is the important one. Three lines of evidence lead to this conclusion. Perhaps the most striking is the experimental evidence of McIver et al. (1926), who produced peritonitis in cats by the intraperitoneal injection of faeces and then killed them 28 hours later. As expected, autopsy showed a generalized peritonitis with distension of the intestines as far down as the colon: however, if at the time of the injection of faeces the pylorus was occluded by ligature, then at autopsy no distension was found. Further evidence is provided by analysis of the gas in the intestine in cases of ileus. This shows, first, the presence of only small quantities of hydrogen sulphide and methane, which would be expected in large quantities if decomposition was the main source; and, secondly, that some 80 per cent. of the gas consists of nitrogen, with rather more $\mathrm{CO}_{2}$ and less $\mathrm{O}_{2}$ than is present in air. These findings are precisely those that would be expected if swallowed air is the source of the gas. The rate at which gases are absorbed from the intestine depends upon their relative tension in the blood and intestinal lumen, and the composition of air is so similar to the blood gases that once the $\mathrm{O}_{2}$ and $\mathrm{CO}_{2}$ have reached equilibrium further absorption takes place only very slowly. McIver et al. (1926) distended loops of cats' intestines with various gases and found that, whereas $\mathrm{O}_{2}$ and $\mathrm{CO}_{2}$ are rapidly absorbed, nitrogen is only taken up slowly and, as would be expected from this, that air is absorbed at approximately the same rate as nitrogen.

The final argument is provided by the clinical evidence that if after operation careful steps are taken to minimize the passage of air into the intestines, then ileus can be largely prevented. This point has been investigated by many workers. Typical results are those of Davis and Hansen (1945), who compared two groups of patients undergoing a variety of abdominal operations, one group being treated by continuous gastric suction after operation, the other not receiving this treatment; they found that only ro per cent. of the treated group became distended after operation, as compared to 29 per cent. of the controls.

This conclusion that the gas in the distended intestines is air implies that it must have been swallowed. At first sight inherently improbable, this is, in fact, what most probably occurs. Paine, Carlson and Wangensteen (1933) observed a series of patients who were treated after operation by continuous gastric suction. They found that on an average $2,000 \mathrm{ml}$. of air per day were aspirated from patients undergoing appendicectomy or hernia repair, 3,000 ml. per day from those having operations on the biliary tract, and $3,500 \mathrm{ml}$. from those having gastric operations. Admittedly obtained from patients with indwelling tubes, these figures nonetheless emphasize the unexpectedly large quantities of air which may be swallowed in the first few days following operation.

The mechanism by which this swallowing takes place has been elucidated by Maddock and his co-workers in two papers (Morris et al., 1947; Maddock et al., 1949) which repay close study. In summary they have shown that the swallowing of air is normally prevented by the cricopharyngeus muscle and that if during inspiration this muscle is relaxed, then air readily passes into the oesophagus, from which it is propelled into the stomach. This process is much accentuated if at the time of swallowing the glottis is closed. Maddock showed that in this process, which he refers to as "airsucking,' the average normal oesophageal pressure of $-6 \mathrm{~cm}$. $/ \mathrm{H}_{2} \mathrm{O}$ is converted to $-3 \mathrm{I} \mathrm{cm} . / \mathrm{H}_{2} \mathrm{O}$, and that practised ' air-suckers' swallowed into their stomachs over $100 \mathrm{ml}$. of air with each suck, whilst subjects who could not belch at will nonetheless swallowed $60 \mathrm{ml}$. of air when making an inspiratory effort against a closed glottis. He further showed that subsequent belching does not remove as much air as is swallowed, so that the repetition of these 
movements can lead to rapid distension of the .stomach.

Many nervous people regularly swallow air and belch when upset and it is a common clinical observation that patients complaining post-operatively of wind also often complain of flatulence, and say that if only they could get the wind up they would feel better. In fact, in their efforts to break wind these patients simply swallow more air, and careful observation shows that many more patients than is commonly realized indulge in this trick after operation, presumably stimulated thereto by the discomfort following surgery.

\section{The Effects of Distension}

Normally if air is swallowed or introduced into the stomach it passes through the intestine very rapidly. First demonstrated by Magnusson (I93I), this has since been confirmed by many workers, notably again Maddock (Maddock et al., 1949), who showed that on the average swallowed air takes some ${ }_{5}$ minutes to reach the caecum and that it appears as flatus in 25 minutes. However, air swallowed after operation will enter not a normal alimentary tract, but one in which peristalsis is markedly inhibited; as a result its passage will be impeded and some degree of distension must occur. Now distension of the intestines, especially if marked, produces a series of effects which tend both to exacerbate the distension and cause further inhibition of gut motility. These effects may be summarized as follows: first, kinking of the intestine is caused, adding some degree of mechanical obstruction; secondly, distension of one part of the small intestine causes inhibition of all types of movement in the rest of the gut, as a result of reflexes passing via the extrinsic nerves of the intestine (Youmans et al., I938); thirdly, over-stretching of the gut musculature further impairs its ability to contract; and, fourthly, there is an increasing interference with the venous return from the distended loops, leading to further troubles. Thus, it causes an increased exudation of plasma into the peritoneal cavity and into the wall and lumen of the gut; it causes an increased secretion and diminished absorption of intestinal juices, leading to their accumulation in the gut, which is so marked a feature of both organic and functional obstruction; and, finally, it threatens the viability of the distended loop, again interfering with its motility and eventually leading to necrosis and perforation.

\section{Additional Factors}

There are several other factors which undoubtedly predispose to the development of ileus. Mecray, Barden and Ravdin (1937) have shown that in hypoprotinaemic dogs the gastric emptying time is delayed and that there is oedema of the gut $\frac{0}{20}$ wall, which may well interfere with its motility, and it is possible that protein deficiency explains the clinical observation that patients in a poor $\stackrel{\varnothing}{\circ}$ nutritional state are particularly prone to the con- $\stackrel{c}{-}$ dition. It is also possible that vitamin deficiencies $\overrightarrow{\vec{F}}$ may interfere with gut motility, Jacques (I95I) suggesting that a deficiency of pantothenic acid $\frac{C}{0}$ may be of importance and claiming excellent results $\frac{\bar{D}}{\overline{0}}$ from its administration in cases of established $\underset{\varnothing}{\Omega}$ ileus. But of far greater importance are the acute $\stackrel{\varrho}{0}$

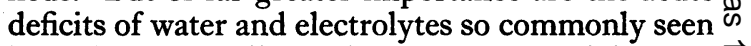
in patients with ileus, their importance lying not $\vec{\circ}$ so much in their being primary aetiological $\overrightarrow{ }$ factors, but in their tendency to predispose to and $\vec{\sigma}^{\circ}$ exacerbate the condition. Most of the current interest centres around the effects of potassium 3 . depletion, but it must not be forgotten that alterations in water and salt balance can influence gut $\underset{\omega}{\omega}$ motility. As long as 20 years ago several workers (Perazzo, 1937) showed that alterations in the plasma concentration of sodium altered gut $\varnothing$ motility, and more recently Marriott (1947) has commented on the greatly delayed gastric emptying time seen in patients with salt depletion.

The possible connection between paralytic ileus and potassium deficiency was first suggested by Randall et al. (1949), who described three typica cases of post-operative potassium deficiency, all $f$ whom, amongst other signs, showed marke chronic ileus. The association between the tuī. conditions is now well recognized. On the one hand, it is a common clinical experience to find that patients with established ileus are hypokalaemic and improve when they receive potassium; and, on the other hand, ileus is a characteristic $\stackrel{\square}{\perp}$ manifestation of potassium depletion (Lans et al., $\overrightarrow{\overrightarrow{\vec{B}}}$ 1952). Some workers have gone so far as to suggest $\frac{3}{3}$ that potassium deficiency is indeed the cause of paralytic ileus. Streeten and Ward McQuaid (1952), in a study of 13 cases, found that during the period of ileus there was an excess urinary loss 3 . of potassium, with a lowering of the plasma con- $\frac{\dot{\sigma}}{3}$ centration, and that during recovery there was a retention of potassium. They suggested that para- $\frac{\rho}{2}$ lytic ileus is due to potassium deficiency, in its turn due to prolonged adrenocortical activity in $\frac{\text { 의 }}{2}$ the face of an inadequate potassium intake. $D$ Streeten and Vaughan Williams (1952) investigated the same point experimentally in dogs and found that the production of a severe intracellular or potassium depletion caused a marked depression $N$ of gut motility, but the conditions produced in $N_{\omega}$ these experiments varied in important respects from those seen clinically.

There is no doubt that potassium depletion $\underset{\mathbb{D}}{\mathbb{D}}$ depresses gut motility, that in some cases of severe $\stackrel{\oplus}{+}$ ileus the administration of this ion is essential to 7 
recovery, and that in any case of prolonged ileus the existence of potassium depletion should always be considered. There is no real evidence, however, that potassium depletion in itself can cause true ileus, though it is without doubt an important subsidiary factor in that by depression of gut motility it may convert a case of severe "wind 'into one of true ileus and seriously prolong and aggravate the established condition.

To summarize, the underlying factor in the aetiology of paralytic ileus is a period of reflex inhibition of gut motility occurring after many operations. Usually this passes off uneventfully, but may not do so if the patient is an air swallower. If only moderate quantities of air are swallowed, nothing worse will ensue beyond so-called postoperative ' wind '; but if excessive air is swallowed or the impairment of gut motility is unduly prolonged, distension will become marked and a vicious circle set up, leading to frank ileus. Potassium depletion, by virtue of its depression of gut motility, is an important subsidiary factor which may precipitate and prolong ileus. In that ileus leads to severe losses of water and electrolytes, the effect of potassium depletion is both progressive and cumulative.

\section{Clinical Features}

The clinical picture of paralytic ileus is too well known to require detailed description. In the early stages distension is the prominent sign, but as the condition progresses vomiting becomes increasingly marked and with it the well-known stigmata of water and electrolyte depletion, until in the end death ensues as a result of peripheral circulatory failure and toxic absorption from the distended, devitalized intestine. Apart from distension, the most significant abdominal sign is the complete silence on auscultation of the abdomen, broken only by an occasional tinkling, high-pitched note resulting from passive movement of the intestines. If necessary, the diagnosis may be confirmed by $\mathrm{X}$-ray, plain films of the abdomen showing a characteristic picture with fluid levels in both small and large intestine. It may be very difficult to decide whether or not the ileus is accompanied by an actual peritonitis, and in the presence of greatly distended intestine careful judgment is needed to determine the significance of tenderness and rebound tenderness, but if these are marked, and particularly if localized, they probably indicate the presence of peritonitis.

On occasion it may be difficult to differentiate between paralytic ileus and plastic peritonitis giving rise to complete or partial organic obstruction of the intestines at multiple sites from fibrinous adhesions. Both conditions tend to develop after an incident of general peritonitis and their clinical picture may be similar. However, ileus usually comes on two to four days afterwards, whilst obstruction due to plastic adhesions develops later, often eight to ro days after the peritonitis. Pain, as opposed to discomfort, is unusual in ileus, but if the obstruction is essentially organic there will generally be some intestinal colic, at least in the early stages, with increased bowel sounds. Further, in organic obstruction radiology will generally show no gas in the large gut, as opposed to its generalized distribution in ileus.

\section{Treatment \\ Prevention}

As previously emphasized, the critical aetiological factor in paralytic ileus is the development of distension. This is much more easily prevented than it is controlled and relieved when once it has developed. Accordingly, in clinical practice the emphasis must be, not on treatment, but on prophylaxis, and particularly the prevention of distension.

Considering first pre-operative measures, excessive purgation during the days immediately prior to operation should be avoided and if the bowel requires emptying a simple enema is preferable. Secondly, ileus is particularly likely to develop in nervous, highly-strung patients, and it is important that such people receive adequate sedation before operation; despite their mild depressant effect on the bowel, barbiturates in suitable doses are invaluable for this purpose. Thirdly, and of greater importance than these simple points, if the intestines are distended they should, whenever possible, be deflated before operation is undertaken. In many cases the nature of the disease will not allow of delay in surgery, but when this is possible great benefit can come from deferring operation whilst the intestine is deflated by suction down a Miller-Abbott or Ryle's tube. It is impossible to lay down rules for how long such treatment should be persevered with, but, provided care is taken to see that there is no progressive, intra-peritoneal inflammatory process and that the patient's fluid and electrolyte equilibrium is maintained, there is usually little or no harm in continuing intestinal suction for several days. There is no doubt that if complete deflation of the intestine can be achieved the operation itself will be much simpler and the patient's recovery smoother.

The anaesthetist also has an important part to play in preventing ileus, as he can, unless careful, allow considerable quantities of air to enter the stomach. Maddock et al. (1949) showed that during the smooth induction of an inhalational anaesthetic only a negligible quantity of air enters the stomach, but in a patient who became obstructed during- 
induction $720 \mathrm{ml}$. of air were aspirated from the stomach. This consequence of an obstructed airway is not generally recognized and is an additional reason for maintaining a clear airway during anaesthesia. Modern anaesthetic techniques also have their special danger, in that under certain circumstances positive pressure anaesthesia can force air down the gullet into the stomach, particularly if positive pressure is applied via a facepiece to a patient receiving muscle-relaxant drugs. Accordingly, every effort should be made to avoid using positive pressure, except down an endotracheal tube. Both these points are well-accepted anaesthetic teaching, but their neglect may lead to serious consequences after operation.

During the course of the operation, to diminish irritation of the peritoneum the intestines should be handled as little and as gently as possible, and care should be taken to minimize soiling of the peritoneum with bowel contents, pus, etc. If it is essential to open the abdomen with distended intestines, whenever possible these should be deflated before the abdomen is closed. This can be done quite safely by inserting a sucker into the gut through a small opening and then evacuating the fluid and gaseous contents by threading the intestine over the sucker. In addition to facilitating closure of the abdomen, there is good clinical evidence that this procedure is of great value in preventing ileus in this type of case, and it is a wise rule never to close the abdomen over distended intestines unless there is some urgent contraindication to this simple manoeuvre.

It is, however, the management after surgery that is of supreme importance in preventing ileus, particularly in cases undergoing major abdominal surgery or with definite peritonitis. The stomach must be kept empty, or at the very least prevented from distension, until such a time as normal motility has returned to the intestines. This can only be done by restricting the fluid intake by mouth, and possibly in addition by carrying out gastric suction, until such time as normal bowel sounds return and the patient passes flatus either spontaneously or with the aid of glycerine suppositories. The restriction of fluids by mouth after major abdominal surgery is so well known as to need no further comment. The place of suction is, however, more controversial. Some authorities recommend gastric suction routinely after all major abdominal operations, whilst others only use it when ileus is impending or established. A compromise between these two views is probably the best. It is wise to start suction immediately after operation in all cases where there is extensive handling of the intestines or where there is definite peritonitis, but in other cases it seems justifiable to watch the patient's progress and then pass a tube if there is any significant distension, if the patient is nauseated or vomits, or shows pronounced aerophagy. Once started, suction should continues until bowel sounds have returned and the oraf. balance shows that fluids are passing on normally into the intestine. If at this stage flatus has not been passed, a glycerine enema or suppositories: will usually produce the desired result. Laxatives are contraindicated, as they may retard the return of normal bowel motility.

This compromise policy may on occasion allowes definite distension, even mild ileus, to develop, but $\overrightarrow{0}$ it saves many patients from the discomforts of $\dot{a}-$ naso-gastric tube and, provided such a tube iș passed promptly if there are any signs of ileus developing, little harm is likely to occur. It seems to make little difference whether the tube is aspirated continuously or intermittently, provided $\omega$ the intervals between aspiration do not exceed oneos hour. If 30 to $60 \mathrm{ml}$. of water are given by mouth directly after aspiration the intermittent methodo shows at once when the stomach starts to emptyo itself, and continuous suction is only required when large quantities of air or fluid must be removed. Some authorities advise that a Miller- $-\mathbb{\infty}$ Abbott tube should be passed into the intestine $\frac{D}{?}$ before operation and used post-operatively for pr-o phylactic aspiration. Save for the purpose \&f relieving pre-operative distension, this see illogical, as it is obviously preferable to remove the. air from the stomach before it has got into theo intestines, and it is not unknown for it to be necessary, with a tube well down in the intestines, to pass a Ryle's tube as well to control distension.

Generally it will only be necessary to restrict $\stackrel{2}{\varrho}$ oral fluids and use gastric suction for two to three days after operation, but during this time the fluid $\frac{0}{3}$ and electrolyte balance must be maintained, usually by giving intravenous fluids in appropriate quantities (Le Quesne, 1957). Of great interest in respect to the prevention of ileus is the question as to 3 whether this intake should contain potassium. If none is given, a potassium deficit must necessarily?. develop, this being accentuated by the potassium diuresis, which occurs in the first 24 to 48 hours after operation. As mentioned, a potassium deficito undoubtedly predisposes to ileus, and it seems illogical to allow such a deficit to develop and theno perhaps later have to treat its results. It is unnecessary and possibly unwise to give potassiumer for the first 24 to 48 hours after operation, but if intravenous fluids must be continued thereafter it ${ }_{\mathrm{c}}$ is quite safe if the usual precautions are taken. $A_{\sigma}$ reasonable intake is $6 \mathrm{~g}$. $\mathrm{KCl}(80 \mathrm{~m} . \mathrm{Eq} \mathrm{K})$ per 240 hours, and this is conveniently given together with $\frac{\bar{\Phi}}{\widetilde{D}}$ the daily ration of salt $(4.5 \mathrm{~g} . ; 80 \mathrm{~m} . \mathrm{Eq} \mathrm{Na})$ in? 21 . of 2.5 per cent. dextrose. It is wise to take at 0 least two hours running in each 0.51 . of thiso 
solution. During the last four years we have given this solution to a very large number of patients. No complications have been found to arise from this treatment and balance studies show that this intake prevents the development of any significant deficit without the risk of a dangerous retention of potassium. It is difficult to prove that by this means many cases of ileus have been prevented, but it is our clear clinical impression that this is so and it certainly prevents the development of severe potassium depletion.

\section{The Established Case}

By application of the measures outlined it should be possible to prevent most patients developing florid ileus with severe distension and fluid losses. Cases will be met with in which the motility of the intestines is unduly slow in returning, who develop some degree of distension and who lose considerable quantities of fluid by suction, but it is usually possible to prevent the development of classical ileus provided suction is persevered with and the fluid intake maintained, with adequate replacement of abnormal losses. Patience is essential in handling these cases. It is preferable to continue 'drip and suction' for 24 hours too long than 24 hours too little, as if too much is given orally too early or the bowels stimulated by purgatives or drugs before peristalsis is re-established the return of normal bowel function can be distinctly retarded. In some cases the long delay of return of bowel function raises the possibility that there may be an organic obstruction, due probably to adhesions. It is probably wise only to operate on such cases when there is strong evidence of organic obstruction and a laparotomy in the hope of finding some situation capable of correction is rarely of benefit. It is in cases of this sort that the possibility of a potassium deficit must be carefully considered, particularly if none has been given. Whilst clinical evidence can suggest most strongly this diagnosis, it should always be confirmed by estimation of the serum potassium concentration, and an E.C.G. may be helpful. If confirmed, the deficit should be replaced in the usual way (see Le Quesne, 1957 , for details of diagnosis and treatment of $\mathrm{K}$ depletion).

The treatment of established ileus is essentially similar to its prophylaxis. In the past many surgeons have advocated the use of active measures designed to stimulate the gut by the use of turpentine or ox-bile enemata, the administration of a spinal anaesthetic, or the use of drugs, such as pitocin or carbachol. In some cases these methods may achieve success, presumably by breaking the vicious circle caused by increasing distension. But such treatment is illogical, usually ineffective, and often positively harmful. The intestines cannot be made to resume normal peristalsis and attempts to do so are more likely to delay than expedite the natural return of function. But recovery can be encouraged by preventing the entry of further air into the intestines, by relieving distension, and by correcting any disturbances in fluid and electrolyte balance.

These objects are achieved by gastro-intestinal suction and by the intravenous administration of fluids. Again there is the question as to whether to use a gastric or Miller-Abbott tube. The protagonists of this latter tube argue that if it passes the pylorus it will deflate the upper coil of the bowel, which will then regain its peristaltic activity and push the tube into the next loop and so on down the bowel. The difficulty lies in getting the tube to pass the pylorus when the alimentary tract is immotile, and even when this has been achieved the tube will not prevent the further swallowing of air. Most surgeons find that a stomach tube passed into the intestine is simpler and just as effective. By this means further entry of air into the intestines can be prevented and, owing to regurgitation, sufficient deflation can be achieved to allow of recovery of peristalsis.

Aside from suction, the most important part of 0 the treatment of established ileus lies in the restoration and maintenance of fluid and electrolyte balance. This involves all the problems connected with the assessment and replacement of preexisting deficits of salt and water, the replacement of observed losses, and the diagnosis and treatment of potassium depletion. These problems lie outside the scope of this paper, but it must be emphasized that, despite the great importance of potassium depletion in ileus, the correct handling of the patient's water and sodium balance remains of paramount importance and demands first consideration.

A further problem arises with respect to the use of sedatives, especially morphia, in the treatment of paralytic ileus. There is still controversy regarding the effect of morphia on the motility of the gut, but it is probable that, in man, it diminishes peristalsis whilst increasing the tone of the intestine. In view of this it is sometimes argued that the drug is contraindicated in patients with ileus, but this is to overlook its powerful central effects, which are essentially beneficial in that they allay apprehension, relieve pain and ensure rest to patients badly in need of all these. The weight of clinical evidence undoubtedly supports the view that morphia is a drug of the utmost value in these patients and that only benefit can come from giving it in adequate dosage, both in the prevention and treatment of paralytic ileus.

Bibliography continued on page 626 
abnormal losses, the making good of her established deficit, blood transfusion and ultimately the giving of her post-operative fluid requirements.

At her second operation recurrence of the disease was found in the terminal ileum and the sigmoid colon was also severely affected. A terminal ileostomy was therefore established, which further increased the need for intravenous fluid replacement.

This infusion was maintained for a total of 17 days and transmitted a total of 501 . of fluid, including blood. There were no complications and the patient was intensely grateful for her vena caval infusion, having had the experience of a conventional superficial vein infusion at the time of her first operation.

\section{Summary}

I. The technique of introducing intravenous fluids direct into the venae cavae by means of a polythene cannula is described.

2. The indications for using this technique and its advantages are discussed.

3. The results are given of a series of cases in which this method has been used.

\section{CARCINOMA OF THE BRONCHUS}

\section{(Postgraduate Medical Journal)}

Price 3s. 9d. post free

\section{INTRODUCTORY}

Maurice Davidson, D.M., F.R.C.P.

THE INCIDENCE AND AETIOLOGY OF PRIMARY CARCINOMA OF THE LUNG

C. E. Drew, M.V.O., F.R.C.S.

\section{MEDICAL ASPECTS}

J. Anderson, M.D., F.R.C.P.

RADIOLOGICAL ASPECTS

G. Simon, M.D., D.M.R.E., F.F.R.
UNUSUAL MANIFESTATIONS

J. Smart, M.D., F.R.C.P.

CYTOLOGICAL EXAMINATION OF THE SPUTUM AND PLEURAL EFFUSION

J. L. Pinniger, D.M., M.R.C.P.

THE SCOPE OF RADIOTHERAPY

Gwen Hilton, D.M.R.E., F.F.R.

SURGERY OF CARCINOMA OF THE BRONCHUS

L. L. Bromley, M.Chir., F.R.C.S.

Published by

THE FELLOWSHIP OF POSTGRADUATE MEDICINE

60, Portland Place, London, W.1

Continued from page 61 I-Paralytic Ileus: L. P. Le Quesne, D.M., F.R.C.S.

\section{BIBLIOGRAPHY}

BISGARD, J. D., and JOHNSON, E. K. (1939), Ann. Surg., IIO, 802.

DAVIS, H. H., and HANSEN, T. M. (1945), Surgery, 17, 492.

DEVINE, J. (1946), Brit. F. Surg., 34, 158.

JACQUES, J. E. (1951), Lancet, ii, 861.

LANS, H. S., STEIN, I. F., and MEYER, K. A. (1952), Surg. Gynec. Obstet., 95, 321 .

LE QUESNE, L. P. (1957), ' Fluid Balance in Surgical Practice,' and ed., Lloyd-Luke (Medical Books) Ltd., London.

MADDOCK, W. G., BELL, J. L., and TREMAINE, M. J. (1949), Ann. Surg., 130, 512.

MAGNUSSON, W. (1931), Acta radiol., 12, 552.

MARRIOTT, H. L. (1947), Brit. med. F., i, 245, 285, 328.

MECRAY, P. M., BARDEN, R. P., and RAVDIN, I. S. (1937), Surgery, i, 53.
MORRIS, C. R., IVY, A. C., and MADDOCK, W. G. (1947), Arch. Surg. (Chicago), 55, IOI.

MCIVER, M. A., BENEDICT, E. B., and CLINE, J. W. (1926), Ibid., 13, 588.

PAINE, J. R., CARLSON, H. A., and WANGENSTEEN, O. H. $N$ (1933), Ұ. Amer. med. Ass., 100, 1910.

PERAZZO, G. (1937), Arch. ital. Chir., 47, 163.

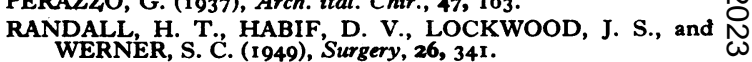

STREETEN, D. H. P., and VAUGHAN WILliaMS, E. M. (1952), F. Physiol. (Lond.), $118,149$.

STREETEN, D. H. P., and WARD-MCQUAID, J. N. (1952), Brit. med. F., 2, 587 .

WAKIM, K. G., and MANN, F. C. (1943), Gastroenterol. 1, 513. YOUMANS, W. B., MEEK, W. J., and HENIN, R. C. (1938), Amer. F. Physiol., 124, 270. 